\title{
Relationship between intraoperative measured parameters of parathyroid gland and pathological patterns in patients with secondary hyperparathyroidism
}

\author{
Xiaoliang Sun ${ }^{1}$, Xiaoqing Zhang ${ }^{2}$, Ling Zhang ${ }^{3}$, Meng Yang ${ }^{1}$, Yao Lu ${ }^{1}$ \\ ${ }^{1}$ Department of General Surgery, China-Japan Friendship Hospital, Beijing, China; ${ }^{2}$ Department of anesthesiology, Peking University Third \\ Hospital, Beijing, China; ${ }^{3}$ Department of Nephrology, China-Japan Friendship Hospital, Beijing, China \\ Contributions: (I) Conception and design: Y Lu; (II) Administrative support: None; (III) Provision of study materials or patients: X Sun; (IV) \\ Collection and assembly of data: X Sun, L Zhang, M Yang; (V) Data analysis and interpretation: X Zhang, L Zhang; (VI) Manuscript writing: All \\ authors; (VII) Final approval of manuscript: All authors. \\ Correspondence to: Yao Lu. Department of General Surgery, China-Japan Friendship Hospital, No. 2 Yinghua East Road, Chaoyang District, Beijing \\ 100029, China. Email: zrluyao@163.com.
}

Background: The hyperplastic patterns of parathyroid glands (PTGs) in secondary hyperparathyroidism (SHPT) are critical for surgical indication and deciding on the approach. Earlier histopathological investigations have suggested the occurrence of an initial increase in the parathyroid cells, with a normal lobular structure (diffuse hyperplasia, DH). After this, the PTGs become hyperplastic with some nodules (nodular hyperplasia, NH). The current study aimed to explore the relationship between the intraoperative measurements of weight, volume, and maximal diameter of dissected PTGs and the histopathological diagnosis of SHPT patients with end-stage renal disease.

Methods: A total of 182 SHPT patients who received parathyroidectomy (PTX) were retrospectively enrolled. Altogether 21 patients were selected as having at least one diffuse polyclonal hyperplasia PTG. Intraoperative measurements of weight, volume, and maximal diameter of dissected PTGs were compared between tissues with $\mathrm{DH}$ and $\mathrm{NH}$.

Results: Intraoperative dissected PTGs were verified histologically. The differences in the intraoperative measurements of weight, volume, maximal diameter, and the combination of the three measurements between the DH and the NH PTGs groups were significant $(\mathrm{P}=0.000)$, and the values of area under the ROC curve (AUCs) were 0.824 (95\% CI: 0.731-0.918), 0.812 (95\% CI: 0.716-0.908), 0.746 (95\% CI: 0.633-0.860), and 0.851 (95\% CI: 0.768-0.935), respectively, with cut-off values of the three parameters being $0.19 \mathrm{~g}, 206.3 \mathrm{~mm}^{3}$, and $10.5 \mathrm{~mm}$, respectively.

Conclusions: The measurement of weight, volume, and maximal diameter of dissected PTGs is a possible alternative to assess the hyperplasia patterns of the dissected PTGs. It is a promising reference for the ultrasound prediction of pathological patterns of PTGs.

Keywords: Secondary hyperparathyroidism (SHPT); parathyroid glands (PTGs); diffused hyperplasia; nodular hyperplasia (NH); parathyroidectomy (PTX)

Submitted Feb 16, 2020. Accepted for publication Nov 01, 2020.

doi: $10.21037 / \mathrm{atm}-20-1643$

View this article at: http://dx.doi.org/10.21037/atm-20-1643

\section{Introduction}

Secondary hyperparathyroidism (SHPT) is a severe complication seen in patients with end-stage renal disease
(ESRD). The etiology of SHPT is excessive synthesis and secretion of intact parathyroid hormone (iPTH) and longterm parathyroid hyperplasia. Elevated iPHT may lead to high-turnover bone disease, interstitial and vascular 
calcifications, and cardiovascular morbidity and mortality. Patients' quality of life is drastically compromised, owing to the high occurrence and recurrence of SHPT (1). The hypersecretion of $\mathrm{iPTH}$ is associated with an increase in the size of the parathyroid glands (PTGs), initially characterized by diffuse hyperplasia (DH) and subsequently by nodular hyperplasia (NH), and eventually adenoma formation at the advanced stage. Some patients develop refractory SHPT when the volume of a hyperplastic PTG is $>500 \mathrm{~mm}^{3}$ or the long diameter of a gland $>1 \mathrm{~cm}$; this strongly suggests nodular transformation. After 5-10 years on dialysis, about $15 \%$ of patients require parathyroidectomy (PTX) for refractory SHPT $(2,3)$. Therefore, histopathological alterations are important for the diagnosis and treatment of SHPT in ESRD patients.

PTX is essential when conservative therapy is no longer effective in controlling the disease, or the patient is refractory to treatment (4). The surgical indications for SHPT patients considering ultrasound (US) parameters in the Chinese, Japanese, and European guidelines are different. There is limited evidence concerning the relationship between the analyzed parameters of the hyperplasia of PTG with the US and histopathological findings (5). However, the US evaluation is highly dependent on the operator's experience and instrument performance. Sometimes ectopic parathyroid hyperplasia is undetectable with the US (retrosternal PTG). The sensitivity of imaging studies usually ranges from $30-80 \%$ (6).

Intraoperative measurement of dissected PTG is a more direct and accurate method, and predictive of pathological results. Furthermore, it may help surgeons differentiate the DH PTGs from NH PTGs when parathyroid autotransplantation is required. Some studies suggested that the increased size, along with the altered quality of the PTG mass, might be responsible for non-controllable SHPT in uremia (7), while the preoperative imaging examinations could not differentiate the DH PTGs from NH PTGs. Therefore, an intraoperative inspection of the dissected PTGs would better analyze the quality of diseased PTGs and predict pathological changes.

The current study aimed to explore the relationship between the parameters of intraoperative dissected PTGs and the final histopathological findings of ESRD patients with SHPT. This preliminary study suggests a method to make intraoperative surgical decisions, and conversely, may offer a reference method for the US evaluation and prediction of pathological patterns in PTGs to decide treatment modality. We present the following article under the STROBE reporting checklist (available at http://dx.doi. org/10.21037/atm-20-1643).

\section{Methods}

\section{Patient selection and study design}

We retrospectively reviewed a total of 182 SHPT patients with ESRD requiring regular renal dialysis who received PTX at the China-Japan Friendship Hospital from February 2017 to April 2018. All patients were enrolled regardless of the success of the PTX surgery. This study was conducted in accordance with the Declaration of Helsinki (as revised in 2013) and was approved by ethics board of China-Japan Friendship hospital (NO.: 2019-SDZL-12). Informed consent was taken from all the patients.

According to histopathological findings, of the 182 patients (a total of 683 PTGs), none were diagnosed as DH in the all dissected PTG samples, while 159 (602 PTGs) were diagnosed as $\mathrm{NH}$. Altogether 23 patients were diagnosed with at least one $\mathrm{DH}$, and two cases were excluded. Of these, one had a second PTX after 15 months. Exploratory surgery found multiple nodules in the right lobe of the thyroid tissue, which, after histopathological examination, were diagnosed as multifocal parathyroid adenomatous hyperplastic nodules with active growth. The case review showed the possibility of parathyroid tissue implantation in the thyroid gland during the first operation as a single parathyroid nodule was detected in the lower lobe of the right thyroid. This case was excluded as the size of the nodule could not be measured. The other case was excluded as the pathological finding of one dissected PTG was inconclusive due to sample quality. To avoid analytic error due to sample size differences, patients whose pathological findings confirmed the existence of both $\mathrm{DH}$ and $\mathrm{NH}$ were selected.

\section{Surgical indications}

The surgical indications for ESRD patients with SHPT in our hospital are (I) persistent elevation of iPTH to values greater than $800 \mathrm{pg} / \mathrm{mL}$; (II) uncontrolled hypercalcemia with hyperphosphatemia or clinical symptoms of SHPT refractory to treatment; (III) resistance to medical therapies; (IV) the evidence of more than one PTG hypertrophy with diameter $\geq 10 \mathrm{~mm}$, and rich in blood flow through powerDoppler images. 
Table 1 Patient characteristics

\begin{tabular}{lc}
\hline Characteristics & Patients $(\mathrm{n}=21)$ \\
\hline Female, $\mathrm{n}(\%)$ & $10(47.6)$ \\
Age (years) & $42.48 \pm 12.09$ \\
Body mass index $\left(\mathrm{kg} / \mathrm{m}^{2}\right)$ & $22.59 \pm 3.42$ \\
Duration of dialysis $(\mathrm{m})$ & $75.5 \pm 41.98$ \\
Hemodialysis modality (hemodialysis/ & $18 / 3$ \\
peritoneal dialysis) & \\
Causes of kidney failure & \\
Glomerulus nephritis, $\mathrm{n}$ & 12 \\
Hypertension, $\mathrm{n}$ & 3 \\
Drug, $\mathrm{n}$ & 1 \\
Kidney stones, $\mathrm{n}$ & 1 \\
Unknown, $\mathrm{n}$ & 4 \\
Patients receiving calcimimetics, $\mathrm{n}(\%)$ & $6(28.6)$ \\
Pre-operative intact parathyroid & $1,688.99 \pm 925.12$ \\
hormone (pg/mL) & $2.41 \pm 0.26$ \\
Preoperative calcium (mmol/L) & \\
Preoperative phosphorus (mmol/L) & \\
\hline
\end{tabular}

\section{Variables and measurements}

All operations were performed by experienced surgeons. Intraoperative measurements of the weight and dimensions of each dissected PTG were recorded by two independent researchers. The volume of each PTG was estimated by using the formula: $\mathrm{a} \times \mathrm{b} \times \mathrm{c} \times \pi / 6 \mathrm{~mm}^{3}$ (where $\mathrm{a}, \mathrm{b}, \mathrm{c}$ are the dimensions of the gland in $\mathrm{mm}$ (6). The measurements and calculations were performed quickly to keep the surgical duration minimum.

\section{Histological classification of PTGs}

Every dissected PTG was examined by two independent pathologists unaware of the results of any variables or the calculation methods. The pattern of parathyroid hyperplasia was classified based on the histological classification of each PTG as DH, NH, or adenomas. Pathological diagnosis was made according to the following criteria: DH contained proliferative parathyroid parenchymal cells forming lobular structures, while early $\mathrm{NH}$ was of a single-cell type, forming nodules lacking fibrous bands. In an advanced stage of NH, the glands exhibited at least single well-circumscribed, encapsulated nodule, each consisting of a single cell type with fat-free accumulation. The final diagnosis in the pathological report was used for correcting the actual number, and accordingly, the volume and weight of the identified PTG.

\section{Data availability}

The datasets generated and analyzed during the current study are available from the corresponding author on reasonable request.

\section{Statistical analysis}

The statistical analysis was performed using SPSS 22.0 version (SPSS Inc., Chicago, IL, USA). Continuous data with normal distribution were presented as means \pm standard deviation (SD). Student's $t$-test was used to compare continuous variables. The non-normally distributed continuous variables were presented as the median and interquartile range (IQR) and compared using the Mann-Whitney U test. Variables included the weight, volume, and maximal diameter of each dissected PTG. Receiver operating characteristic (ROC) curve analysis was performed to depict the area under the ROC curve (AUC) to better evaluate the diagnostic value of the selected variates.

\section{Results}

A total of 80 dissected PTGs from 21 patients were removed surgically, of which 77 were of parathyroid origin as proved by pathological examination, and the remaining 3 were lymph nodes. Of pathologically verified 77 PTGs, 2 PTGs were in 1 patient, 3 PTGs in 6, 4 PTGs in 13, and 5 PTGs in 1 patient. Histological evaluation verified a total of 42 dissected PTGs as NH, 33 as DH, and 2 were adenomas. The demographic, clinical, and laboratory results of 21 patients are summarized in Table 1.

Of 77 dissected PTGs, preoperative US detected 49 PTGs (63.6\%) (1 PTG in seven patients, 2 PTGs in five, 3 PTGs in four, and 4 PTGs in five patients). According to surgical indications in our hospital, only 25 PTGs were detected by the US with a diameter $\geq 1 \mathrm{~cm}$, which were verified as NH PTGs by pathological findings. US indicated surgical dissection of PTGs accounting for $59.5 \%$ of all NH PTGs.

The differences in the volume, weight, and maximal 
Table 2 Intraoperative measurement of parameters between diffuse hyperplasia and nodular hyperplasia groups

\begin{tabular}{|c|c|c|c|c|}
\hline Parameters & \multicolumn{3}{|c|}{ Pathology of parathyroid gland } & $P$ value \\
\hline Weight (g) & $0.100(0.045,0.175)$ & $0.450(0.223,0.965)$ & $\mathrm{n} 1=8.100 ; \mathrm{n} 2=6.700$ & 0.000 \\
\hline Volume $\left(\mathrm{mm}^{3}\right)$ & $90.0(36.0,188.0)$ & $316.5(186.0,792.0)$ & $\mathrm{n} 1=7,560.0 ; \mathrm{n} 2=8,400.0$ & 0.000 \\
\hline Maximal diameter (mm) & $9.0(7.5,10.5)$ & $13.0(9.8,17.0)$ & $\mathrm{n} 1=30.0 ; \mathrm{n} 2=30.0$ & 0.000 \\
\hline
\end{tabular}

The non-normally distributed continuous variables were presented as median and interquartile range (IQR) and compared using the MannWhitney $\mathrm{U}$ test. * $\mathrm{P}$ value $<0.05$ when comparing diffuse hyperplasia and nodular hyperplasia groups.

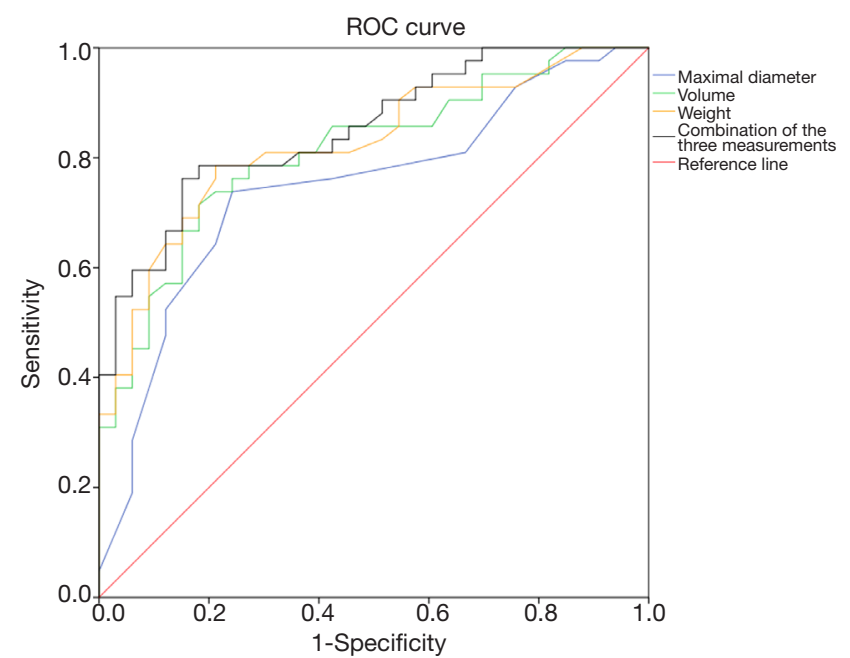

Figure 1 ROC curves for intraoperative measurement of weight, volume, the maximal diameter, and the combination of the three measurements of dissected PTGs. The AUCs of weight, volume, the maximal diameter, and the combination of the three measurements of intraoperative dissected PTGs were $0.824(95 \%$ CI: $0.731-0.918), 0.812$ (95\% CI: 0.716-0.908), 0.746 (95\% CI: 0.633-0.860), and 0.851 (95\% CI: 0.768-0.935), respectively. Cut-off values of weight, volume, and the maximal diameter of intraoperative dissected PTGs were $0.19 \mathrm{~g}$ (sensitivity $78.6 \%$, specificity $78.8 \%$ ), $206.3 \mathrm{~mm}^{3}$ (sensitivity $71.4 \%$, specificity $81.8 \%$ ) and $10.5 \mathrm{~mm}$ (sensitivity $73.8 \%$, specificity $75.8 \%$ ), respectively. ROC, receiver operating characteristic; AUC, area under the ROC curve; CI, confidence interval; PTGs, parathyroid glands.

diameter of the PTGs measured intraoperatively between $\mathrm{DH}$ and $\mathrm{NH}$ groups were significant $(\mathrm{P}=0.000)$ (Table 2).

The AUCs for weight, volume, maximal diameter and the combination of the three measurements of intraoperative dissected PTGs were 0.824 (95\% CI: 0.731-0.918), 0.812 (95\% CI: $0.716-0.908), 0.746$ (95\% CI: $0.633-0.860)$, and 0.851 (95\% CI: 0.768-0.935), respectively (Figure 1). Cut-off values of these parameters were $0.19 \mathrm{~g}$ (sensitivity $78.6 \%$, specificity $78.8 \%$ ), $206.3 \mathrm{~mm}^{3}$ (sensitivity $71.4 \%$, specificity $81.8 \%$ ), and $10.5 \mathrm{~mm}$ (sensitivity $73.8 \%$, specificity $75.8 \%)$, respectively.

\section{Discussion}

Data from the Dialysis and Transplantation Registration System revealed that there are about 200 million patients on dialysis in China duet to the increasing prevalence of SHPT (8). The Japanese Society for Dialysis Therapy reported that about $10 \%$ of patients receive PTX 10 years after dialysis and $30 \%$ of SHPT patients require surgical treatment when the dialysis has been lasted for more than 20 years (9).

The estimation of hyperplastic patterns of PTGs in SHPT patients is critical when deciding surgical indications (10). The hypersecretion of iPTH is associated with PTG enlargement and histological changes. The pathological changes of PTGs are characterized by initial DH to early nodularity, subsequent $\mathrm{NH}$, and finally, to the formation of a single nodular gland (11). NH of PTG exceeds the extreme point during pathological progression and is the key issue for the failure of medical treatment in SHPT patients (5). However, only limited evidence exists concerning the relationship between the US measurement and pathological results, with inconclusive predictive value. The sensitivity is lower in SHPT than in primary hyperparathyroidism. An earlier pathological investigation found almost all glands weighing more than $500 \mathrm{mg}$ were nodular hyperplastic glands (12). Other studies revealed larger than $300 \mathrm{~mm}^{3}$ volume of PTG, and more than $8-\mathrm{mm}$ diameter could predict $\mathrm{NH}$ (11-13).

However, a US examination is subjective, depending heavily on the operator's experience. US cannot detect all the PTGs, and sometimes the ectopic hyperplasia of 
the PTG is undetectable. In the current study, of the 77 intraoperative dissected PTGs, US could found only $49(63.6 \%)$. The actual volume of the glands estimated by US was lower than the weight values found by the direct measurement. Tominaga et al. reported that a gland volume of $330 \mathrm{~mm}^{3}$ estimated by US was equivalent to a gland weight of $500 \mathrm{mg}$ measured at the time of surgery. The researchers postulated that the underestimation of US might be due to the unsuitable use of the formula or inaccuracy from the US itself (12). The sensitivity of US detection varies widely, and even with a combination of neck US and scintigraphy, bilateral surgical exploration with identification of all glands is required (6).

Intraoperative measurement of dissected PTGs is a more direct and accurate method of assessing the relationship between actual size and weight of PTGs and pathological findings. In China, delayed diagnosis of SHPT is common as a large number of ESRD patients are ignorant of this disease and receive treatment when severe complications such as severe bone deformity, short height, or severe heterotopic ossification occur. Medications are effective in the initial treatment in alleviating symptoms of bone pain and lowering PTH. However, many patients discontinue the treatment as symptoms of hypercalcemia, and gastrointestinal intolerance are common; the expensive medications are difficult to afford, and medical treatment is ultimately ineffective for these patients as SHPT progresses to an advanced stage. Thus, these patients need to resort to a surgical option. This study demonstrated that $87 \%$ (159/182) of surgical patients were diagnosed as NH of all dissected PTGs, with all patients having at least one PTG diagnosed as NH by pathology.

In this study, we performed intraoperative measurements of the dissected PTGs. The AUCs for weight, volume, and maximal diameter of the dissected PTGs were 0.824 , 0.821 , and 0.746 , respectively. According to the ROC results, the sensitivity and specificity of intraoperative direct measurement of PTGs during PTX are encouraging. This could be helpful for recognizing and predicting histological characters of PTGs intraoperatively along with influencing surgical decisions.

Currently, there are three surgical approaches for PTX, namely, subtotal PTX (sPTX), total PTX (tPTX), and tPTX with auto-transplantation (tPTX + AT). The guidelines set by the kidney disease outcomes quality initiative (K/DOOI) and most experts recommend sPTX and tPTX + AT for SHPT, but there is a lack of consensus on the best operative management (14). The major disadvantage with sPTX is the difficulty in re-exploration of the thyroid bed for recurrent disease, with potential injury to the recurrent laryngeal nerve (15). Therefore, surgeons are more inclined to perform tPTX + AT. According to our study, intraoperative analysis of the weight, volume, and maximal diameter of the dissected PTGs could provide critical information about the histological character of dissected tissues and help in selecting the suitable PTGs for auto-transplantation. A dissected PTG weighing more than $0.19 \mathrm{~g}$, with a volume larger than $206.3 \mathrm{~mm}^{3}$, and maximal diameter longer than $10.5 \mathrm{~mm}$ has a higher possibility of being $\mathrm{NH}$ and is unsuitable for auto-transplantation.

The current US criteria used in our hospital are reliable and similar to the result of the maximal diameter calculated in our preliminary study. However, the intrinsic disadvantage of US renders it insufficient to reflect other characteristics of the PTGs. Although the weight and volume of the PTGs could not be measured using two dimensional US, these are more effective in discriminating DH and NH PTGs as shown in this study. In our country, most US reports are only measuring the length and width of PTGs. Therefore, intraoperative measurement is more accurate and direct in evaluating the pathological patterns of the hyperplasia PTGs.

The strength of the current study includes: first, by intraoperative real-time measurement of the actual weight, volume, and maximal diameter of the dissected PTGs, we could find a close correlation between these parameters and postoperative final pathological results. This is highly important in guiding surgeons on decision making. This is especially imperative for patients in China since most of the ESRD patients with SHPT are in the advanced stage requiring surgical intervention. If DH PTGs could be identified early during operation, the performance of tPTX + AT could lower the recurrence rate and enhance patients' quality of life. Besides, by simultaneously analyzing all three parameters, a more accurate and comprehensive standard could be established to predict the pathological character of dissected PTG. Moreover, intraoperative recognition and measurement of PTGs would facilitate its preoperative detection and more accurate measurement by the US. Since the imaging technique has significantly progressed in recent times, US evaluation is widely used in the preoperative diagnosis of SHPT. With the development of high-resolution three-dimensional US and more experimental resources invested in this field, we hope that the specificity and sensitivity would increase markedly. By establishing a discriminant database with an enlarged 
sample size of patients receiving PTX, and correlating the measurement data with the US diagnosis, the preoperative evaluation with US could better differentiate and predict the histological characteristic of the PTGs, and make diagnosis and treatment more precise on the targeted patients.

There are some limitations to the current study. Firstly, this is a retrospective study, and the indication bias for surgery could hardly be avoided; besides, the patient enrollment period was short, and the sample size was relatively small. The results might not be generalized to other dialysis patients, as those in our single tertiary center are mostly at the end-stage of the disease with severe symptoms and delayed surgical opportunity. We investigated only 21 patients from 182 SHPT patients since most of our patients were at an advanced stage, and the pathological findings confirmed all dissected PTGs as NH. Further studies are warranted with a larger sample size, enrolling more patients with DH PTGs to verify the current study and formulate a more precise criterion.

In conclusion, we provided a feasible and reliable method for intraoperative differentiation of the pathological patterns of parathyroid hyperplasia nodules by measuring weight, volume, and maximal diameter of the dissected nodules. This would help the surgeons in making decisions during operation and enhancing patients' quality of life significantly. Moreover, intraoperative recognition and measurement of PTGs would provide a study basis for the early detection and evaluation of pathological features with the advancement of US instruments.

\section{Acknowledgments}

Funding: This study was supported by Beijing Municipal Science \& Technology Commission (No. Z191100006619014).

\section{Footnote}

Reporting Checklist: The authors have completed the STROBE reporting checklist. Available at http://dx.doi. org/10.21037/atm-20-1643

Data Sharing Statement: Available at http://dx.doi. org/10.21037/atm-20-1643

Conflicts of Interest: All authors have completed the ICMJE uniform disclosure form (available at http://dx.doi. org/10.21037/atm-20-1643). The authors have no conflicts of interest to declare.

Ethical Statement: The authors are accountable for all aspects of the work in ensuring that questions related to the accuracy or integrity of any part of the work are appropriately investigated and resolved. This study was conducted in accordance with the Declaration of Helsinki (as revised in 2013) and was approved by Ethics Board of China-Japan Friendship hospital (NO.: 2019-SDZL-12). Informed consent was taken from all the patients.

Open Access Statement: This is an Open Access article distributed in accordance with the Creative Commons Attribution-NonCommercial-NoDerivs 4.0 International License (CC BY-NC-ND 4.0), which permits the noncommercial replication and distribution of the article with the strict proviso that no changes or edits are made and the original work is properly cited (including links to both the formal publication through the relevant DOI and the license). See: https://creativecommons.org/licenses/by-nc-nd/4.0/.

\section{References}

1. Li JG, Xiao ZS, Hu XJ, et al. Total parathyroidectomy with forearm auto-transplantation improves the quality of life and reduces the recurrence of secondary hyperparathyroidism in chronic kidney disease patients. Medicine (Baltimore) 2017;96:e9050.

2. Vulpio C, Bossola M, Di Stasio E, et al. Histology and immunohistochemistry of the parathyroid glands in renal secondary hyperparathyroidism refractory to vitamin D or cinacalcet therapy. Eur J Endocrinol 2013;168:811-9.

3. Lau WL, Obi Y, Kalantar-Zadeh K. Parathyroidectomy in the Management of Secondary Hyperparathyroidism. Clin J Am Soc Nephrol 2018;13:952-61.

4. Cunningham J, Locatelli F, Rodriguez M. Secondary hyperparathyroidism: pathogenesis, disease progression, and therapeutic options. Clin J Am Soc Nephrol 2011;6:913-21.

5. Jäger MD, Serttas M, Beneke J, et al. Risk-factors for nodular hyperplasia of parathyroid glands in sHPT patients. PLoS One 2017;12:e0186093.

6. Fang L, Tang B, Hou D, et al. Relationship between parathyroid mass and parathyroid hormone level in hemodialysis patients with secondary hyperparathyroidism. BMC Nephrol 2015;16:82.

7. Lewin E, Olgaard K. Influence of parathyroid mass on the regulation of PTH secretion. Kidney Int Suppl 
2006;(102):S16-21

8. Tian W, He Q, Jiang K, et al. Expertise consensus on clinical practice of hyperparathyroidism secondary to chronic renal failure. Chinese Journal of Practical Surgery 2016;36:481-6.

9. Nakai S, Suzuki K, Masakane I, et al. Overview of regular dialysis treatment in Japan (as of 31 December 2008). Ther Apher Dial 2010;14:505-40.

10. Onoda N, Kurihara S, Sakurai Y, et al. Evaluation of blood supply to the parathyroid glands in secondary hyperparathyroidism compared with histopathology. Nephrol Dial Transplant 2003;18 Suppl 3:iii34-iii37.

11. Tominaga Y, Tanaka Y, Sato K, et al. Histopathology, pathophysiology, and indications for surgical treatment of renal hyperparathyroidism. Semin Surg Oncol 1997;13:78-86.

12. Tominaga Y, Matsuoka S, Sato T, et al. Clinical

Cite this article as: Sun X, Zhang X, Zhang L, Yang M, Lu Y. Relationship between intraoperative measured parameters of parathyroid gland and pathological patterns in patients with secondary hyperparathyroidism. Ann Transl Med 2021;9(2):99. doi: 10.21037/atm-20-1643 features and hyperplastic patterns of parathyroid glands in hemodialysis patients with advanced secondary hyperparathyroidism refractory to maxacalcitol treatment and required parathyroidectomy. Ther Apher Dial 2007;11:266-73.

13. Matsuoka S, Tominaga Y, Sato T, et al. Relationship between the dimension of parathyroid glands estimated by ultrasonography and the hyperplastic pattern in patients with renal hyperparathyroidism. Ther Apher Dial 2008;12:391-5.

14. National Kidney Foundation. K/DOQI clinical practice guidelines for bone metabolism and disease in chronic kidney disease. Am J Kidney Dis 2003;42:S1-S201.

15. Yoo J, Young JEM. Secondary hyperparathyroidism: surgical. In: Khan AA, Clark OH, editors. Handbook of parathyroid diseases: a case-based practical guide. 1st edition. London: Springer, 2012:159-80. 